Published in final edited form as:

Nat Photonics. 2017 October ; 11(10): 657-663. doi:10.1038/s41566-017-0001-7.

\title{
Giant light-harvesting nanoantenna for single-molecule detection in ambient light
}

\author{
Kateryna Trofymchuk ${ }^{1}$, Andreas Reisch ${ }^{1}$, Pascal Didier ${ }^{1}$, François Fras ${ }^{2}$, Pierre Gilliot ${ }^{2}$, \\ Yves Mely ${ }^{1}$, and Andrey S. Klymchenko, ${ }^{1, *}$ \\ ${ }^{1}$ Université de Strasbourg, Laboratoire de Biophotonique et Pharmacologie, UMR 7213 CNRS, \\ Faculté de Pharmacie, 74, Route du Rhin, F-67401 ILLKIRCH, France \\ ${ }^{2}$ CNRS, IPCMS, UMR 7504, F-67034 Strasbourg, France
}

\begin{abstract}
Here, we explore the enhancement of single molecule emission by polymeric nano-antenna that can harvest energy from thousands of donor dyes to a single acceptor. In this nano-antenna, the cationic dyes are brought together in very close proximity using bulky counterions, thus enabling ultrafast diffusion of excitation energy ( $\leq 30 \mathrm{fs}$ ) with minimal losses. Our 60-nm nanoparticles containing $>10,000$ rhodamine-based donor dyes can efficiently transfer energy to 1-2 acceptors resulting in an antenna effect of $\sim 1,000$. Therefore, single Cy5-based acceptors become 25 -fold brighter than quantum dots QD655. This unprecedented amplification of the acceptor dye emission enables observation of single molecules at illumination powers $\left(1-10 \mathrm{~mW} \mathrm{~cm}^{-2}\right)$ that are $>10,000$-fold lower than typically required in single-molecule measurements. Finally, using a basic setup, which includes a 20X air objective and a sCMOS camera, we could detect single Cy5 molecules by simply shining divergent light on the sample at powers equivalent to sunlight.
\end{abstract}

Detection of single fluorescent molecules requires a dedicated microscopy setup with strong excitation power density from 100 to $5,000 \mathrm{~W} \mathrm{~cm}^{-2}$ due to the limited brightness of fluorescent dyes.1,2 The use of much lower excitation power would not only decrease photo-damage3,4 and auto-fluorescence background, but also enable utilization of inexpensive light sources5 important for high-throughput screening and diagnostics assays. Interestingly, fluorescent nanoparticles (NPs) that are many-fold brighter than single dyes can be detected even using a smartphone-based device.6 Could a low power excitation similar to the ambient light intensity be used for single molecule detection? The typical

\footnotetext{
Users may view, print, copy, and download text and data-mine the content in such documents, for the purposes of academic research, subject always to the full Conditions of use:http://www.nature.com/authors/editorial_policies/license.html\#terms

Correspondence and requests for materials should be addressed to A.S.K. andrey.klymchenko@unistra.fr; Tel: +33 368854255. Author contributions

A.S.K. proposed the concept; A.S.K. and K.T. designed the experiments; K.T. performed most of the experiments and data analysis. A.R. performed electron microscopy and helped with donor NP design and some data analysis. P.D. and F.F. performed time-resolved anisotropy measurements. A.S.K. helped with single-particle microscopy. A.R., Y.M., P.G. and A.S.K. contributed materials/analysis tools. A.S.K. and K.T. wrote the manuscript.

Competing financial interests

European patent application was filed under no. 17305763.9.

Reprints and permissions information is available online at www.nature.com/reprints.
} 
power density of sunlight at the earth surface in the visible range is $1-5 \mathrm{~mW} \mathrm{~cm}^{-2}$ for a 10 $\mathrm{nm}$ bandwidth, 7 which is similar to that used in fluorometers or plate readers but $10^{4}-10^{6}$ times lower than that used in single molecule microscopy. Recent works suggested that metallic nanostructures, so-called plasmonic nano-antennas, can amplify the excitation and emission of single molecules.8-12 Between two gold NPs, a 100-300-fold amplification could be achieved.13,14 Larger amplification values were also reported, but only when the fluorophore was initially poorly emissive or quenched.14,15 To detect single molecules under ambient light, we need to achieve at least 1,000-fold amplification of the signal coming from a high performance non-quenched fluorophore, such as cyanine 5 (Cy5). A promising direction is to use the light-harvesting concept realized by Nature in photosynthetic centres, 16 where multiple strongly communicating donors can efficiently collect and deliver the light energy to a single acceptor using Förster Resonance Energy Transfer (FRET).17 Chemists developed highly efficient FRET systems that use conjugated polymers, 18-20 dendrimers,21,22 multi-porphyrin arrays, 23 micellar NPs,24-26 polymer NPs, 27 dye assemblies, 28,29 metal-organic frameworks, 30 or nucleic acids. 31 However, the obtained amplification of the acceptor has never reached 1,000 so far,32 being limited by self-quenching of donors at high local concentration and/or inefficient donor-donor coupling. A promising light-harvesting platform is dye-loaded polymer nanoparticles. $33 \mathrm{We}$ showed recently that encapsulation of multiple dyes inside polymer NPs using bulky counterions minimized dye self-quenching and produced fluorescence switching (blinking) of up to 500 dyes per particle.34 It was explained by unprecedented coupling of encapsulated dyes due to exciton migration (excitation energy transfer, EET), so that a single dark species could quench the whole dye ensemble. Here, we hypothesized that much larger dye ensembles coupled by efficient EET inside NPs could generate giant light-harvesting antenna for amplifying the fluorescence of a single dye. This hypothesis was successfully realized using cationic rhodamine dyes encapsulated in a poly(methyl methacrylate-comethacrylic acid) (PMMA-MA) matrix using bulky fluorinated counterions. These NPs of $\sim 60 \mathrm{~nm}$ diameter can generate unprecedented 1,000-fold amplification of a single Cy5 derivative located inside the particles. This result enabled for the first time observation of single molecules at room temperature using excitation equivalent to sunlight.

\section{Results}

The nano-antenna was designed based on PMMA-MA NPs of 40-60 nm encapsulating large amounts of energy donor dyes (Fig. 1). The hydrophobic fluorinated anion tetrakis(pentafluorophenyl)borate (F5-TPB) was used to facilitate encapsulation of cationic rhodamine B (R18) and minimize its self-quenching. 34 The increase in dye loading from 0.1 to $30 \mathrm{wt} \%$ (with respect to the mass of the polymer) somewhat increased the NPs hydrodynamic diameter (Supplementary Table 1), although transmission electron microscopy (TEM) suggested relatively small size ( $44 \pm 2 \mathrm{~nm}$ ) of NPs at $30 \mathrm{wt} \%$ loading, which corresponded to $\sim 5,900$ dyes per NP.

The fluorescence quantum yields (QY) of the obtained NPs decreased with the dye loading but remained remarkably high $(\sim 0.30)$ even at $30 \mathrm{wt} \%$ loading (Fig. 2a). This result corroborated with relatively small changes in the absorption and emission spectra of NPs (Supplementary Fig. 1). The F5-TPB counterion probably functions as a spacer between the 
charged dyes that prevent their self-quenching through pi-stacking.34 Time-resolved measurements revealed that the mean fluorescence lifetime decreased with donor dye loading (Fig. 2b), which correlated well with the QY decrease. The estimated radiative rates remained stable, whereas non-radiative rates gradually increased (Fig. 2b), and short-lived components appeared (Supplementary Table 2). Nevertheless, these non-radiative processes were not fast enough to prevent efficient fluorescence of NPs.

High dye loadings are expected to decrease the inter-fluorophore distances and thus favour dye-dye EET,35,36 which can be evidenced by a fluorescence anisotropy decrease. 37 Indeed, the highest dye loading (30 wt $\%$ ) produced a nearly 100-fold drop in fluorescence anisotropy (Fig. 2a). At this loading the average distance between encapsulated dyes should be $\sim 1 \mathrm{~nm}$, which is far below the 4.6-nm Förster radius of homo-FRET for R18/F5-TPB (Supplementary Table 3), thus favouring fast EET. We then investigated the emission anisotropy decay using the pump-probe technique. For low dye loading ( $1 \mathrm{wt} \%$ of R18/F5TPB), the anisotropy decreased only marginally during the first 10 ps (Fig. 2c), indicating that EET in this system is slow and requires a much longer time to reach the steady-state anisotropy value. By contrast, at high dye loading (30 wt $\%$ ) the anisotropy decayed to zero already within $1 \mathrm{ps}$ (Fig. 2c). A bi-exponential fit revealed $30 \mathrm{fs}(45 \%)$ and $600 \mathrm{fs}(55 \%)$ components, the former being limited by the resolution of our setup ( $60 \mathrm{fs}$ pulse width). The EET process is thus exceptionally fast and can involve thousands of dyes within their emission lifetime (fluorescence lifetime / anisotropy decay time $=3 \times 10^{3}-5 \times 10^{4}$ ).

Next, we introduced DiD, a hydrophobic Cy5 derivative (Fig. 1), acting as an energy acceptor for rhodamine B inside NPs. Being encapsulated at $0.02 \mathrm{wt} \%$, DiD displayed a high fluorescence quantum yield of $0.77 \pm 0.04$. For NPs containing $30 \mathrm{wt} \%$ of donor dyes, an increase in the acceptor concentration resulted in a rapid growth of acceptor emission, accompanied by a drop of the donor emission, indicating a FRET process (Fig. 2d). Based on donor emission, the FRET efficiency reached $28 \pm 5 \%$ already for $0.004 \mathrm{wt} \%$ of the acceptor (Fig. 2e), which corresponded to 1.2 acceptors per 44-nm particle (Poisson distribution of acceptors is shown in Supplementary Table 4). Time-resolved measurements revealed a picosecond raise component at the acceptor channel (Supplementary Fig. 2 and Table 5), which suggested fast FRET rates, increasing with acceptor loading from $10^{9}$ to $5 \times 10^{9} \mathrm{~s}^{-1}$ (Fig. 2e). The kinetics data indicate that FRET efficiency can reach values of $67 \%$ at $0.004 \mathrm{wt} \%$ acceptor. As overall population of dyes within this particle ( 5,900 dyes) exhibits $28 \%$ FRET efficiency, $67 \%$ corresponds to FRET efficiency from $\sim 2,340$ dyes to a single acceptor (Supplementary Table 6).

For a constant concentration of the acceptor $(0.004 \mathrm{wt} \%)$ inside NPs, an increase in the donor loading produced a significant growth in FRET efficiency (Fig. 2f) and acceptor relative intensity (Supplementary Fig. 3a,b). Assuming that no EET occurs among donors, negligibly low FRET efficiency (4-6\%) should be observed independently from the donor loading (Fig. 2f, Supplementary Note 1 and Table 7). Thus, at high donor loading, fast EET likely ensures efficient FRET from thousands of donors to a single acceptor.

Owing to efficient FRET, our NPs should behave like light-harvesting nano-antenna. To quantify the antenna effect (AE), we recorded and analysed the excitation spectra of our 
FRET NPs at the emission wavelength of the acceptor (Supplementary Fig. 3c,d). At low donor dye loading, no antenna effect was observed ( $\mathrm{AE} \sim 1$ ), whereas at higher donor loadings, AE increased rapidly reaching $555 \pm 30$ for $44-\mathrm{nm}$ NPs at $30 \mathrm{wt} \%$ (Fig. $2 \mathrm{~g}$ ). These values correlated well with the antenna effect calculated from FRET efficiency and expected number of donor and acceptor dyes per NP (Fig. 2f).31,38 By contrast, theoretical estimations assuming no EET suggested 3-6 fold lower AE values.

Since EET ensures efficient FRET through donor-acceptor distances above the Förster radius, we investigated the influence of the size of $30 \mathrm{wt} \%$ dye-loaded NPs on the nanoantenna performance. Due to the dependence of NP size on the polymer charge, 39 we varied the $\mathrm{pH}$ of the phosphate buffer used in nanoprecipitation, to change the protonation state of carboxylate in PMMA-MA polymer. According to dynamic light scattering (DLS), a decrease in $\mathrm{pH}$ from 9.0 to 5.8 produced an increase in NP size from 30 to $230 \mathrm{~nm}$, while preserving a good polydispersity (Table 1). TEM confirmed this tendency, but revealed smaller NP sizes, ranging from 30 to $110 \mathrm{~nm}$ (Fig. 3a).

Independently of the size, the QY of $30 \mathrm{wt} \%$ dye-loaded NPs remained high (0.27-0.31, Table 1) and their mean fluorescence lifetime (1.6-1.9 ns) and decay components (Supplementary Table 8 ) were practically invariant. Moreover, according to wide-field fluorescence microscopy of NPs immobilized on a glass surface, the single particle brightness increased with size (Fig. 3b, Supplementary Fig. 4a), so that the largest NPs (NPs110) were 31-fold brighter than the smallest ones (NPs30) (Table 1), in line with estimations based on QY and the size of NPs measured by TEM. Finally, the anisotropy values were close to zero for all particle sizes (Table 1), indicating efficient EET.Then, we prepared nano-antennas of different sizes containing $30 \mathrm{wt} \%$ of donors with varied amount of acceptor corresponding to donor/acceptor ratios 1,000 and 10,000 (0.02 and $0.002 \mathrm{wt} \%$, respectively). For both ratios, an increase in NP size increased the contribution of the acceptor emission and the FRET efficiency (Fig. 4a, Supplementary Fig. 4b,c). The antenna effect also increased with NP size, especially for NPs with donor/acceptor ratio of 10,000 (Fig. 4b). Remarkably, for NPs60 and NPs65 (containing 1.3 and 1.7 acceptors per NP, respectively) the antenna effect reached $910 \pm 100$ and $1150 \pm 150$, respectively (Fig. 4b). To our knowledge, these are the highest values of FRET-based amplification of dye emission reported to date. We also verified whether our nano-antennas can amplify fluorescence of acceptor dye directly at the particle surface, using a less hydrophobic analogue of DiD, Cy5C2. According to titration data, Cy5-C2 binds well NPs45, (Supplementary Fig. 5a,b), and the bound dye exhibits good QY (0.28 \pm 0.04$)$. In case of $45 \mathrm{~nm}$ NPs loaded at $30 \mathrm{wt} \%$ with R8/F5-TPB, a strong FRET with nearly equal intensity of acceptor and donor bands was observed for a donor-acceptor ratio of 1,000 (Fig. 4c). Remarkably, based on the excitation spectra (Supplementary Fig. 5c,d), the antenna effect for NPs of different sizes was systematically >200 (Fig. 4d). Thus, our nano-antennas can strongly amplify the acceptor emission also at their surface, although the antenna effect is weaker than for the acceptor inside NPs.

Under wide-field TIRF microscope, immobilized control NPs60 without acceptors appeared as bright spots at the donor channel and as dim spots at the acceptor channel. In the presence of statistically 1.3 acceptors per particle (Supplementary Table 9), the emission in the 
acceptor channel became comparable or brighter than that in the donor channel (Fig. 5a). Analysis of the intensities in the two channels confirmed these observations (Supplementary Fig. 6a), suggesting that the emission of 1-2 acceptors inside the nano-antennas was comparable to the emission of thousands of donor dyes. To confirm the number of acceptors per NP, we studied the intensity traces of the acceptor through direct excitation at $642 \mathrm{~nm}$. For nano-antennas of different sizes formulated to contain statistically 1-2 acceptors (Supplementary Table 9), the most frequent number of bleaching steps until complete signal loss was 1 (Fig. 5b), indicating that, indeed, nano-antennas contained usually only a single acceptor per particle.

Remarkably, to obtain similar acceptor intensities, the excitation through nano-antennas NPs60 at $532 \mathrm{~nm}$ required $\sim 1,000$-fold lower laser power than direct excitation of the acceptor at $642 \mathrm{~nm}$ (Figure 5c,d, Supplementary Figs. 6a, 7). The amplification factor (antenna effect), calculated based on excitation power and acceptor emission at these two conditions was found to increase with the particle size (Fig. 5e). For NPs60, the obtained amplification factor was $\sim 1,040 \pm 100$, which was in good agreement with the antenna effect measured from the excitation spectra. Owing to this giant amplification factor, the brightness of 1-2 acceptors inside NPs60 was 25-fold higher than that of quantum dots QD655 excited at $532 \mathrm{~nm}$ with the same power (Fig. $5 \mathrm{c}, \mathrm{d}$ ). This is an exceptional performance of single Cy5 dyes, taking into account that the extinction coefficient of QD655 at $532 \mathrm{~nm}$ is $2.4 \times 10^{6}$ $\mathrm{M}^{-1} \mathrm{~cm}^{-1}$ (data from the provider) and QY is close to unity.

Finally, using our NPs60 nano-antenna, we could observe single molecule events, such as one-step bleaching of the Cy5 (DiD) acceptor at different excitation power densities, reaching extremely low values of $1 \mathrm{~mW} \mathrm{~cm}^{-2}$ (Fig. 5f,g). The fraction of bleaching events (Fig. 5g) and the on-time before photo-bleaching (Supplementary Fig. 8) correlated well with the laser power. Remarkably, in $14-25 \%$ of beaching events, the acceptor bleaching was accompanied by one-step growth of the donor emission (Fig. 5f,g). This opposite behaviour of donor and acceptor is typically observed in single molecule FRET measurements using one donor and acceptor dyes, 40 but here FRET takes place between 13,000 donors and 1-2 acceptors. The amplitude of the donor recovery constituted $12-32 \%$ of the average brightness of NPs without acceptor (dependent on the excitation power, see Supplementary Fig. 6b,c), which is equivalent to 1,600-4,200 donor dyes turning on after the acceptor photobleaching. When 1,600 donor dyes (observed for $100 \mathrm{~mW} \mathrm{~cm}^{-2}$ ) transfer all energy to a single acceptor the estimated antenna effect is 800 , which is in good agreement with the experimental data.

Next, we tested whether our nano-antennas enable detection of single molecules using a simple microscopy setup where the excitation is provided by directly shining light on the sample at powers equivalent to sunlight (Supplementary Fig. 9). The measured power density of direct sunlight (at midday, 19 October, 2016, Strasbourg) through the excitation filter $527 / 50 \mathrm{~nm}$ was $24 \mathrm{~mW} \mathrm{~cm}^{-2}$. In our setup, we applied an artificial white light source providing $15 \mathrm{~mW} \mathrm{~cm}^{-2}$ through the same filter. The fluorescence of immobilized NPs60 nano-antennas containing statistically $1.3 \mathrm{Cy} 5$ (DiD) dyes was collected using either 20X air or 60X oil immersion objectives and detected using a scalable complementary metal-oxide semiconductor (sCMOS) camera (Fig. 5h). According to overlaid images of donor (green) 
and acceptor (red) channels, these NPs displayed significant acceptor emission (appear in yellow-red), in contrast to control NPs without acceptor that appeared in green (Fig. 5i). Moreover, after 5-min illumination with our artificial light source, the former NPs lost the acceptor emission, probably due to acceptor bleaching, and became similar to the control NPs. Strikingly, using the 20X air objective, we were able to record one-step bleaching events in the acceptor channel (in 30-40 \% of observed NPs), corresponding to single Cy5 dye molecules (Fig. 5j). By contrast, the control NPs displayed much lower intensities in the acceptor channel without abrupt bleaching steps. The on-time before photobleaching in this case was similar to that recorded using TIRF microscope at $1 \mathrm{~mW} \mathrm{~cm}{ }^{-2}$ (Supplementary Fig. 8). Moreover, the total number of collected photons per molecule before photo-bleaching was nearly the same as that obtained in the TIRF setup using both direct and FRET-based excitation (Fig. 5k), being in agreement with the literature data for Cy5.41 These data confirm that the signal recorded under sunlight mimicking conditions corresponds to single Cy5 molecules.

\section{Discussion}

The values of the antenna effect reported in the literature for FRET-based systems vary in the range of 3-100. For instance, in DNA-origami containing intercalated donor and acceptor dyes the AE value reached 12.31 Nanocrystals of difluoroboron chromophores at high D/A ratio, which were claimed to be one of the most efficient light-harvesting systems, had an AE value of 28-29.42 Similar values of AE (31) were reported for dye-doped-polymer NPs loaded with coumarin 123 (donor) and Nile Red (acceptor) dyes.43 Our recent report showed that this value can be increased to up to 200 in ionic NPs using R18 and bulky counterions, 38 though we did not provide direct proof for amplification using singlemolecule microscopy. The highest amplification of dye emission reported to date was achieved using plasmonic nano-antennas, where the dye was placed between two gold nanoparticles with help of DNA origami.14 In this case, AE reached 306. Thus, AE of $\sim 1,000$ achieved in the present work goes beyond those reported for other light-harvesting systems and plasmonic nanostructures. The unique efficiency of our system relies on two key factors. The first one is the high quantum yield of $>10^{4}$ donor dyes encapsulated within our polymeric nano-antenna at $30 \mathrm{wt} \%$ loading, which ensures that a large part of the excitation energy is delivered to the acceptor with minimal energy losses. The second factor is the exceptionally fast EET within the donor dyes on the time scale $\leq 30 \mathrm{fs}$, which ensures efficient delivery of the excitation energy from the whole particle to the donors located within Förster distance from the acceptor. Therefore, thousands of donors can transfer energy to single acceptors through distances beyond the Förster radius. Using the fastest and the slowest time components of the anisotropy decay, we roughly estimated that the number of dyes participating in EET during the emission lifetime can be remarkably large, namely between $3 \times 10^{3}$ and $5 \times 10^{4}$. Direct measurements of the FRET rate suggested that in our NPs $\sim 2,340$ dyes can transfer $67 \%$ of their energy to a single acceptor, whereas single acceptor quenching events can be associated with a fluorescence turn on equivalent to $\geq 1,600$ donor dyes. Though one could explain this phenomenon by ultrafast Förster/Dexter homo-transfer mechanisms, recent works on light-harvesting systems propose coherent energy transfer 44,45 for conjugated polymers, 46,47 photosynthetic systems, 48,49 and dye 
assemblies, 28 which could also take place in our nano-antenna. The coherent energy transfer in our nano-antennas could be favored by the very short inter-fluorophore distances $(\sim 1 \mathrm{~nm})$ at $30 \mathrm{wt} \%$ dye loading, controlled by F5-TPB counterion.

The giant amplification achieved here enables detection of single molecules at remarkably low illumination powers $\left(1-10 \mathrm{~mW} \mathrm{~cm}^{-2}\right)$ that are $>10,000$-fold lower than in typical single molecule measurements. Here, it was realized at room temperature using soft materials, whereas previous low-power single-molecule detection was made at $1.8 \mathrm{~K}$ inside a crystal.50 Moreover, our nano-antennas enable detection of single molecules using a simple sCMOScamera-based imaging setup under ambient sunlight-like conditions, which opens the way to single molecule photography. Finally, we also show that the emission of single acceptors can be amplified up to 200-fold at the surface of these nano-antennas, which will enable ultrasensitive detection of molecules in solution at very low excitation powers.

\section{Methods}

Materials

Poly (methyl methacrylate-co-methacrylic acid) (PMMA-MA, 1.3\% methacrylic acid, Mn $\sim 15000, \mathrm{Mw} \sim 34000$ ), acetonitrile (anhydrous, 99.8\%), rhodamine B octadecyl ester perchlorate ( $>98.0 \%$ ), lithium tetrakis(pentafluorophenyl)borate ethyl etherate were purchased from Sigma-Aldrich. DiD oil (1,1'-dioctadecyl-3,3,3',3'tetramethylindodicarbocyanine perchlorate) (Cy5) and quantum dots QD655 were purchased from Life-Technologies. Sodium phosphate monobasic (>99.0\%, Sigma-Aldrich) and sodium phosphate dibasic dihydrate ( $>99.0 \%$, Sigma-Aldrich) were used to prepare $20 \mathrm{mM}$ phosphate buffers at pH 5.8-9.0. Milli-Q water (Millipore) was used in all experiments.

\section{Synthesis}

Rhodamine B octadecyl ester trakis(penta-fluorophenyl)borate (R18/F5) was synthesized by ion exchange and purified by column chromatography as described previously.34 1,1'Diethyl-3,3,3',3'-tetramethylindodicarbocyanine iodide (Cy5-C2) was synthesized as described elsewhere.51

\section{Nanoparticle Preparation}

Stock solutions of the polymer in acetonitrile were prepared at a concentration of $2 \mathrm{mg} \mathrm{mL}^{-1}$ containing different amounts of R18/F5-TPB ( 0.1 to $30 \mathrm{wt} \%$ relative to the polymer). $50 \mu \mathrm{L}$ of the polymer solutions were then added quickly using a micropipette and under shaking (Thermomixer comfort, Eppendorf, 1,000 rpm) to $450 \mu \mathrm{L}$ of $20 \mathrm{mM}$ phosphate at $21^{\circ} \mathrm{C}$. The nanoparticle suspension was then quickly diluted 5-fold with the phosphate buffer $20 \mathrm{mM}$, pH7.4. For preparation of FRET nanoparticles, different concentrations of DiD (from 0.001 wt $\%$ to $0.04 \mathrm{wt} \%$ relative to the polymer) were added to the acetonitrile solution of polymer containing desired concentrations of R18/F5-TPB, and the particles were prepared as described above. To attach FRET acceptor at the particle surface, an aliquot of Cy5-C2 solution in DMSO was added to initially prepared suspension of nanoparticles followed by 5 min incubation. Preparation of NPs of different sizes was achieved by varying the $\mathrm{pH}$ of phosphate buffer at the first dilution. 


\section{Nanoparticle characterization}

Measurements for the determination of the size of nanoparticles were performed on a Zetasizer Nano ZSP (Malvern Instruments S.A.). The mean value of the diameter of the size distribution per volume was used for analysis. Absorption spectra were recorded on a Cary 4000 scan UV-visible spectrophotometer (Varian), excitation and emission spectra were recorded on a FluoroMax-4 spectrofluorometer (Horiba Jobin Yvon) equipped with a thermostated cell compartment. For standard recording of fluorescence spectra, the excitation wavelength was set to $530 \mathrm{~nm}$. The fluorescence spectra were corrected for detector response and lamp fluctuations. To calculate FRET efficiency based on fluorescence spectra, a classical equation was used: $E_{F R E T}=1-\frac{I_{D-A}}{I_{D}}$, where $\mathrm{I}_{\mathrm{D}}$ is the integral donor intensity and $\mathrm{I}_{\mathrm{D}-\mathrm{A}}$ the integral intensity of the donor in the presence of the acceptor. Measurement of fluorescence anisotropy was performed at $20^{\circ} \mathrm{C}$ with a Fluorolog spectrofluorometer (Horiba Jobin Yvon). Excitation wavelength was set to $530 \mathrm{~nm}$ and detection to $585 \mathrm{~nm}$. Each measurement of the anisotropy value corresponds to an average over 10 subsequent measurements of $0.1 \mathrm{~s}$ each. The anisotropy value (r) was expressed as $\mathrm{r}$ $=\left(I_{\|}-I_{\perp}\right) /\left(I_{\|}+2 I_{\perp}\right)$, where $I_{\|}$and $I_{\perp}$ are fluorescence intensities measured in the plane parallel and perpendicular, respectively, to the plane of excitation. To measure the anisotropy value of Cy5-C2 the excitation wavelength was $620 \mathrm{~nm}$ and emission wavelength $655 \mathrm{~nm}$. For standard recording of excitation spectra, the emission wavelength was set to $700 \mathrm{~nm}$. These spectra were corrected for the lamp intensity. Amplification factor of the acceptor emission (antenna effect, AE) was then expressed as the ratio of the maximal excitation intensity of the donor to that of the acceptor with correction from the emission of the donor dyes at $700 \mathrm{~nm}(1)$ :

$$
A E=\frac{I_{D-\mathrm{FRET}}^{e x}-I_{D}^{e x} * f}{I_{A-\mathrm{FRET}}^{e x}}
$$

Where $I_{D-\mathrm{FRET}}^{e x}$ and $I_{A-\mathrm{FRET}}^{e x}$ are the maximal excitation intensities of donor and acceptor in FRET NPs, respectively; $I_{D}^{e x}$ and $I_{A}^{e x}$ are the excitation intensities at the wavelengths of excitation maximum of donor and acceptor in NPs without acceptors, respectively; $f$ is the correction factor calculated as the ratio of maximum emission intensity of donor for FRET

NPs to that for NPs without acceptor dyes $\left(f=\frac{I_{D-\mathrm{FRET}}^{e m}}{I_{D}^{e m}}\right)$.

The value of the antenna effect was also estimated based on the FRET efficiency using the following equation: $\mathrm{AE}=\left(\mathrm{n}_{\mathrm{D}} \times \varepsilon_{\mathrm{D}} \times \mathrm{E}\right) /\left(\mathrm{n}_{\mathrm{A}} \times \varepsilon_{\mathrm{A}}\right)$, where $\mathrm{nD}$ and $\mathrm{nA}$ are the numbers of donors and acceptors, respectively, per particle, $\varepsilon \mathrm{D}$ and $\varepsilon \mathrm{A}$ are the extinction coefficients of donors and acceptors, respectively, and E is the FRET efficiency. Quantum yields of NPs were calculated using Rhodamine 101 in ethanol as a reference (QY=1.0) with an absorbance of 0.01 at $530 \mathrm{~nm} .52 \mathrm{QYs}$ of an acceptor molecule DiD in PMMA-MA matrix (0.02 wt \%) and $\mathrm{C} 5-\mathrm{C} 2$ at the NP surface were measured using $\mathrm{DiD}$ in methanol $(\mathrm{QY}=0.33)$ as a reference.53 


\section{Fluorescence lifetime measurements}

Time-resolved fluorescence measurements were performed with the time correlated singlephoton counting (TCSPC) technique using the excitation pulses at $480 \mathrm{~nm}$ provided by a pulse-picked frequency-doubled Ti-sapphire laser (Tsunami, Spectra Physics) pumped by a Millenia X laser (Spectra Physics). The emission was collected through a polarizer set at the magic angle and an 8-nm band-pass monochromator (Jobin-Yvon H10) at $582 \mathrm{~nm}$ for R18/F5-TPB (donor) and $665 \mathrm{~nm}$ for DiD (acceptor). The instrumental response function was recorded with a polished aluminium reflector. Time-resolved intensity data were treated with the SPCImage software based on residual minimization (RM) technique.54 Radiative $\left(\mathrm{k}_{\mathrm{r}}\right)$ and non-radiative $\left(\mathrm{k}_{\mathrm{nr}}\right)$ rate constants were calculated through QY and mean lifetime of NPs.55 Rate of FRET process was calculated from the negative (raise) component of the emission decay of acceptors excited through FRET.

\section{Time-resolved anisotropy}

For the time-resolved anisotropy measurements, we used an amplified Ti:sapphire laser that produces ultrashort pulses (100 fs) at a repetition rate of $100 \mathrm{kHz} .60 \mathrm{fs}$ linearly polarized pulses centred at $520 \mathrm{~nm}(\Delta \lambda=12 \mathrm{~nm})$ were obtained by means of an optical parametric amplifier (OPA). The pump's power density was around $120 \mathrm{~W} \mathrm{~cm}^{-2}$ for all the measurements. Ultrashort continuum probe pulses were generated in a sapphire crystal (500-800 nm). The normalized differential transmission $\left(\frac{\Delta T(t)}{T}\right)$ of a 60 fs linearly polarized probe (parallel or perpendicular to the pump polarization) centred around $580 \mathrm{~nm}$ $(\Delta \lambda=13 \mathrm{~nm})$ was measured as a function of the pump-probe delay time

$(\mathrm{t}): \frac{\Delta T(t)}{T}=\frac{I^{p}(t)-I^{0}(t)-I_{f l}^{p}}{I^{0}(t)}$, where $P^{P}(t)$ and $I^{O}(t)$ are the intensities of the transmitted probe with and without pump and $I_{f l}^{p}$ is the fluorescence intensity generated by the pump. A monochromator coupled to a liquid nitrogen cooled CCD (charge-coupled device, Princeton Instrument) was used for detection. The time-resolved anisotropy, $I(t)$, was calculated using the following relation (3):

$$
r(t)=\frac{\left(\frac{\Delta T_{/ /}(t)}{T_{/ /}}-\frac{\Delta T_{\perp}(t)}{T_{\perp}}\right)}{\left(\frac{\Delta T_{/ /}(t)}{T_{/ /}}+2 \frac{\Delta T_{\perp}(t)}{T_{\perp}}\right)}
$$

where // and $\perp$ denotes for a probe beam with a linear polarization parallel and perpendicular with respect to the linear polarization of the pump beam. The decay curves were fitted with a bi-exponential function and the analysis took into account the laser pulse duration.

\section{Transmission electron microscopy (TEM)}

Carbon-coated copper-rhodium electron microscopy grids with a 300 mesh (Euromedex, France) were surface treated with a glow discharge in amylamine atmosphere $(0.45 \mathrm{mbar}, 4$ - $4.5 \mathrm{~mA}, 22 \mathrm{~s}$ ) in an Elmo glow discharge system (Cordouan Technologies, France). Then, 
$5 \mu \mathrm{L}$ of the solution of NPs at $0.04 \mathrm{~g} / \mathrm{L}$ were deposited onto the grids and left for $2 \mathrm{~min}$. The grids were then treated for $1 \mathrm{~min}$ with a $2 \%$ uranyl acetate solution for staining. They were observed with a Philips CM120 transmission electron microscope equipped with a LaB6 filament and operating at $100 \mathrm{kV}$. Areas covered with nanoparticles of interest were recorded at different magnifications on a Peltier cooled CCD camera (Model 794, Gatan, Pleasanton, CA). Image analysis was performed using the Fiji software.

\section{Fluorescence Microscopy}

For single particle fluorescence microscopy measurements, the NPs were immobilized on glass surfaces on which a polyethylenimine (PEI) layer was initially adsorbed. The solutions of NPs were diluted 5,000, 2,000, 1,000 and 500 times for NPs30, NPs45, NPs60, NPs65 and NPs 110 correspondingly. $400 \mu \mathrm{L}$ of these solutions per $\mathrm{cm}^{2}$ were then brought in contact with the PEI covered glass for 15 min, followed by extensive rinsing with Milli-Qwater. The surfaces were left in Milli-Q water during microscopy.

Single particle measurements were performed in the TIRF (Total Internal Reflection Fluorescence) mode on a homemade wide-field setup based on an Olympus IX-71 inverted microscope with a high-numerical aperture (NA) TIRF objective (Apo TIRF 100X, oil, NA 1.49, Olympus). A $532 \mathrm{~nm}$ diode laser (Cobolt Samba 100) and a $642 \mathrm{~nm}$ diode laser (Spectra-Physics Excelsior 635) were used to excite the samples. The $532 \mathrm{~nm}$ laser power density was set to $1-100 \mathrm{~mW} \mathrm{~cm}{ }^{-2}$ (light power divided by the illumination spot size of $\sim 10^{-4} \mathrm{~cm}^{2}$ ) by using a polarizer and a half-wave plate $(532 \mathrm{~nm})$. For direct excitation of acceptor Cy5 (DiD), the $642 \mathrm{~nm}$ laser was used with an intensity of $100 \mathrm{~W} \mathrm{~cm}^{-2}$. The fluorescence signal was recorded with an EMCCD (electron multiplying charge-coupled device) (ImagEM Hamamatsu) (0.106 $\mu \mathrm{m}$ pixel size) using the Micro-Manager software. The exposure time was set to $30.53 \mathrm{~ms}$ per image frame. To enable two channel images WVIEW GEMINI image splitting optics were used with the following filter set: dichroic 640 nm (Semrock FF640-FDi01-25x36), bandpass filters 593 20 nm (Semrock FF01-593/40-25) and $685 \pm 20 \mathrm{~nm}$ (Semrock FF02-685/40-25) were used to image R18/F5-TPB and Cy5, respectively. Single particle analysis was performed using the Fiji software: particle locations were detected through a Fiji routine applied to a projection (maximum intensity) of 1,000 frames. After the automatic background subtraction, the mean intensities of circular regions of interest with a diameter of 8 pixels around the found particle locations were then measured within $1 \mathrm{sec}$ integration time (33 frames). The on-time of the acceptor was measured at the single particle level as the time in the emissive state until one-step beaching event. Gain in the donor intensity and drop in the acceptor intensity were measured at the single-particle level as the difference between the intensities before and after single photobleaching step. At least three image sequences $(245$ pixel $\times 245$ pixel $)$ per condition were analysed with, on average, 500-700 particles per sample. All errors correspond to s.e.m. based on at least three measurements.

Then fluorescence intensity in photons per second $\left(n_{d e t}\right)$ was calculated for EMCCD camera as (4): 


$$
n_{\mathrm{det}}=\frac{\left(I_{\text {output }}-I_{\mathrm{dark}}\right) * f}{\text { Gain }_{\text {analog }} * \operatorname{Gain}_{\mathrm{EM}} * \frac{\mathrm{QE}}{100}}
$$

and for sCMOS camera as (5):

$$
n_{\text {det }}=\frac{\left(I_{\text {output }}-I_{\text {dark }}\right) * f}{\frac{\mathrm{QE}}{100}}
$$

where $I_{\text {output }}$ and $I_{\text {dark }}$ are the output signal intensity with and without illumination, respectively, $f$ is a conversion factor in electrons per count (according to manufacturers $f_{E M-C C D}=5.8$ and $\left.f_{C M O S}=0.48\right)$, Gain $_{\text {analog }}$ and Gain $_{E M}$ are the gain sets on the camera, and $Q E$ is a conversion efficiency of photons to electrons in \%.

To calculate the total emitted photons per NP ( $\left.\mathrm{n}_{\mathrm{emit}}\right), \mathrm{I}_{\text {output }}$ was integrated over the on-time (time before fluorophore photobleaching) that gave the total detected photons before the photobleaching ( $\left.\mathrm{n}_{\text {tot.det }}\right)$. Then, $\mathrm{n}_{\text {emit }}$ was calculated based on $\mathrm{n}_{\text {tot,det }}$ and their fraction captured by the objective lens using (6):56

$$
n_{\text {emit }}=\frac{2 * n_{\text {tot.det }}}{1-\sqrt{1-\left(\frac{N A}{n}\right)^{2}}}
$$

where NA is a value of the numerical aperture of the lens and $\mathrm{n}$ is a refraction index of the immersion medium.

The amplification factor (antenna effect) of acceptor emission at the single-particle level was determined using (4):

$$
A F=\frac{I_{A}^{532 n m}}{I_{A}^{642 n m}} \times \frac{P^{642 n m}}{P^{532 n m}}
$$

where $I_{A}^{532 n m}$ and $I_{A}^{642 n m}$ are the mean intensities of acceptors under excitation at 532 and $642 \mathrm{~nm}$, respectively, and $P^{532 \mathrm{~nm}}$ and $P^{642 \mathrm{~nm}}$ are laser powers at the corresponding wavelengths.

\section{Microscopy mimicking ambient sunlight excitation}

The sunlight power density $\left(24 \mathrm{~mW} \mathrm{~cm}{ }^{-2}\right)$ was recorded at midday on 19 October, 2016, Strasbourg region, using a Handheld Laser Power Meter, 1917-R and Semrock band-pass filter $527 \mathrm{~nm}(50 \mathrm{~nm}$ bandwidth). The artificial white light mimicking sunlight was provided by a Cold light source from Zeiss, type KL 1500 LCD. The sample was illuminated from the top $\sim 2 \mathrm{~cm}$ from the divergent light source output through the same 527-nm filter, which 
corresponded to $15 \mathrm{~mW} \mathrm{~cm}{ }^{-2}$ power density at the sample (light power divided by the illumination spot size of $\sim 1 \mathrm{~cm}^{2}$ ). Single-molecule imaging was done using a Nikon Ti-E inverted microscope using CFI Plan Apo 20X air (NA = 0.75) and CFI Plan Apo 60X oil $(\mathrm{NA}=1.4)$ objectives and a Hamamatsu Orca Flash 4 camera. Donor channel was recorded through a $600 \mathrm{~nm}$ band-pass filter ( $50 \mathrm{~nm}$ bandwidth, Semrock), while the acceptor channel used a $647 \mathrm{~nm}$ long-pass filter (Semrock). Data were recorded and analysed using NIS Elements and Fiji software, respectively. The photo of setup is provided in the supplementary information (Supplementary Fig. 9).

\section{Data availability}

The data that support the plots within this paper and other findings of this study are available from the corresponding author upon reasonable request.

\section{Supplementary Material}

Refer to Web version on PubMed Central for supplementary material.

\section{Acknowledgements}

This work was supported by the European Research Council ERC Consolidator grant BrightSens 648528. We thank to C. Ruhlmann from FRISBI platform (ANR-10-INBS-05) for help with electron microscopy. K.T. was supported by a fellowship from the Ministre de la Recherche (France).

\section{References}

1. Holzmeister P, Acuna GP, Grohmann D, Tinnefeld P. Breaking the concentration limit of optical single-molecule detection. Chem Soc Rev. 2014; 43:1014-1028. [PubMed: 24019005]

2. Sauer, M., Hofkens, J., Enderlein, J. Handbook of Fluorescence Spectroscopy and Imaging: From Ensemble to Single Molecules. Wiley-VCH; 2011.

3. Magidson, V., Khodjakov, A. Methods in Cell Biology. Sluder, Greenfield, Wolf, David E., editors. Vol. 114. Academic Press; 2013. p. 545-560.

4. Dixit R, Cyr R. Cell damage and reactive oxygen species production induced by fluorescence microscopy: effect on mitosis and guidelines for non-invasive fluorescence microscopy. Plant $\mathrm{J}$. 2003; 36:280-290. [PubMed: 14535891]

5. Gerhardt I, Mai LJ, Lamas-Linares A, Kurtsiefer C. Detection of Single Molecules Illuminated by a Light-Emitting Diode. Sensors. 2011; 11:905-916. [PubMed: 22346610]

6. Wei QS, et al. Fluorescent Imaging of Single Nanoparticles and Viruses on a Smart Phone. ACS Nano. 2013; 7:9147-9155. [PubMed: 24016065]

7. Prakash, HPGJ. Solar energy : fundamentals and applications. New Delhi: Tata McGraw-Hill; 2000.

8. Novotny L, van Hulst N. Antennas for light. Nat Photonics. 2011; 5:83-90.

9. Su L, et al. Super-resolution Localization and Defocused Fluorescence Microscopy on Resonantly Coupled Single-Molecule, Single-Nanorod Hybrids. ACS Nano. 2016; 10:2455-2466. [PubMed: 26815168]

10. Kinkhabwala A, et al. Large single-molecule fluorescence enhancements produced by a bowtie nanoantenna. Nat Photonics. 2009; 3:654-657.

11. Garcia-Parajo MF. Optical antennas focus in on biology. Nat Photonics. 2008; 2:201-203.

12. Tinnefeld P. Single-molecule detection: Breaking the concentration barrier. Nat Nanotechnol. 2013; 8:480-482. [PubMed: 23770809]

13. Acuna GP, et al. Fluorescence Enhancement at Docking Sites of DNA-Directed Self-Assembled Nanoantennas. Science. 2012; 338:506-510. [PubMed: 23112329] 
14. Puchkova A, et al. DNA Origami Nanoantennas with over 5000-fold Fluorescence Enhancement and Single-Molecule Detection at 25 mu M. Nano Lett. 2015; 15:8354-8359. [PubMed: 26523768]

15. Yuan HF, Khatua S, Zijlstra P, Yorulmaz M, Orrit M. Thousand-fold Enhancement of SingleMolecule Fluorescence Near a Single Gold Nanorod. Angew Chem-Int Edit. 2013; 52:1217-1221.

16. Scholes GD, Fleming GR, Olaya-Castro A, Van Grondelle R. Lessons from nature about solar light harvesting. Nat Chem. 2011; 3:763-774. [PubMed: 21941248]

17. Gartzia-Rivero L, Banuelos J, Lopez-Arbeloa I. Excitation energy transfer in artificial antennas: from photoactive materials to molecular assemblies. Int Rev Phys Chem. 2015; 34:515-556.

18. Tian ZY, Yu JB, Wu CF, Szymanski C, McNeill J. Amplified energy transfer in conjugated polymer nanoparticle tags and sensors. Nanoscale. 2010; 2:1999-2011. [PubMed: 20697652]

19. Yeo H, Tanaka K, Chujo Y. Effective Light-Harvesting Antennae Based on BODIPY-Tethered Cardo Polyfluorenes via Rapid Energy Transferring and Low Concentration Quenching. Macromolecules. 2013; 46:2599-2605.

20. Thomas SW Iii, Joly GD, Swager TM. Chemical sensors based on amplifying fluorescent conjugated polymers. Chem Rev. 2007; 107:1339-1386. [PubMed: 17385926]

21. Galindo JF, et al. Dynamics of Energy Transfer in a Conjugated Dendrimer Driven by Ultrafast Localization of Excitations. J Am Chem Soc. 2015; 137:11637-11644. [PubMed: 26122872]

22. Adronov A, Frechet JMJ. Light-harvesting dendrimers. Chem Commun. 2000:1701-1710.

23. Yang J, Yoon MC, Yoo H, Kim P, Kim D. Excitation energy transfer in multiporphyrin arrays with cyclic architectures: towards artificial light-harvesting antenna complexes. Chem Soc Rev. 2012; 41:4808-4826. [PubMed: 22659941]

24. Chadha G, Yang QZ, Zhao Y. Self-assembled light-harvesting supercomplexes from fluorescent surface-cross-linked micelles. Chem Commun. 2015; 51:12939-12942.

25. Sun HC, et al. Micelle-Induced Self-Assembling Protein Nanowires: Versatile Supramolecular Scaffolds for Designing the Light-Harvesting System. ACS Nano. 2016; 10:421-428. [PubMed: 26634314]

26. Peng H-Q, et al. Artificial Light-Harvesting System Based on Multifunctional Surface-CrossLinked Micelles. Angew Chem-Int Edit. 2012; 51:2088-2092.

27. Bhattacharyya S, Jana B, Patra A. Multichromophoric organic molecules encapsulated in polymer nanoparticles for artificial light harvesting. Chemphyschem. 2015; 16:796-804. [PubMed: 25600650]

28. Winiger CB, Li SG, Kumar GR, Langenegger SM, Haner R. Long-Distance Electronic Energy Transfer in Light-Harvesting Supramolecular Polymers. Angew Chem-Int Edit. 2014; 53:13609_ 13613.

29. Lin HZ, et al. Collective Fluorescence Blinking in Linear J-Aggregates Assisted by Long-Distance Exciton Migration. Nano Lett. 2010; 10:620-626. [PubMed: 20043661]

30. Son HJ, et al. Light-harvesting and ultrafast energy migration in porphyrin-based metal-organic frameworks. J Am Chem Soc. 2013; 135:862-869. [PubMed: 23249338]

31. Woller JG, Hannestad JK, Albinsson B. Self-Assembled Nanoscale DNA-Porphyrin Complex for Artificial Light Harvesting. J Am Chem Soc. 2013; 135:2759-2768. [PubMed: 23350631]

32. Peng H-Q, et al. Biological Applications of Supramolecular Assemblies Designed for Excitation Energy Transfer. Chemical Reviews. 2015; 115:7502-7542. [PubMed: 26040205]

33. Reisch A, Klymchenko AS. Fluorescent Polymer Nanoparticles Based on Dyes: Seeking Brighter Tools for Bioimaging. Small. 2016; doi: 10.1002/smll.201503396

34. Reisch A, et al. Collective fluorescence switching of counterion-assembled dyes in polymer nanoparticles. Nat Commun. 2014; 5:4089. [PubMed: 24909912]

35. Genovese D, et al. Energy transfer processes in dye-doped nanostructures yield cooperative and versatile fluorescent probes. Nanoscale. 2014; 6:3022-3036. [PubMed: 24531884]

36. Trofymchuk K, et al. Exploiting Fast Exciton Diffusion in Dye-Doped Polymer Nanoparticles to Engineer Efficient Photoswitching. J Phys Chem Lett. 2015; 6:2259-2264. [PubMed: 26266601] 
37. Colby KA, et al. Electronic Energy Migration on Different Time Scales: Concentration Dependence of the Time-Resolved Anisotropy and Fluorescence Quenching of Lumogen Red in Poly(methyl methacrylate). J Phys Chem A. 2010; 114:3471-3482. [PubMed: 20170138]

38. Shulov I, et al. Fluorinated counterion-enhanced emission of rhodamine aggregates: ultrabright nanoparticles for bioimaging and light-harvesting. Nanoscale. 2015; 7:18198-18210. [PubMed: 26482443]

39. Reisch A, Runser A, Arntz Y, Mely Y, Klymchenko AS. Charge-Controlled Nanoprecipitation as a Modular Approach to Ultrasmall Polymer Nanocarriers: Making Bright and Stable Nanoparticles. ACS Nano. 2015; 9:5104-5116. [PubMed: 25894117]

40. Roy R, Hohng S, Ha T. A practical guide to single-molecule FRET. Nat Methods. 2008; 5:507516. [PubMed: 18511918]

41. Zhao Q, Young IT, de Jong JGS. Photon budget analysis for fluorescence lifetime imaging microscopy. J Biomed Opt. 2011; 16 086007-086007-086016.

42. Chen PZ, et al. Light-Harvesting Systems Based on Organic Nanocrystals To Mimic Chlorosomes. Angew Chem-Int Edit. 2016; 55:2759-2763.

43. Patra A, Jana B, Bhattacharyya S. Functionalized Dye Encapsulated Polymer Nanoparticle Attached with BSA Scaffold as Efficient Antenna Materials for Artificial Light Harvesting. Nanoscale. 2016; doi: 10.1039/C6NR05201K

44. Beljonne D, Curutchet C, Scholes GD, Silbey RJ. Beyond Förster Resonance Energy Transfer in Biological and Nanoscale Systems. J Phys Chem B. 2009; 113:6583-6599. [PubMed: 19331333]

45. Collini E. Spectroscopic signatures of quantum-coherent energy transfer. Chem Soc Rev. 2013; 42:4932-4947. [PubMed: 23417162]

46. Collini E, Scholes GD. Coherent Intrachain Energy Migration in a Conjugated Polymer at Room Temperature. Science. 2009; 323:369-373. [PubMed: 19150843]

47. Hwang I, Scholes GD. Electronic Energy Transfer and Quantum-Coherence in $\pi$-Conjugated Polymers. Chem Mater. 2011; 23:610-620.

48. Engel GS, et al. Evidence for wavelike energy transfer through quantum coherence in photosynthetic systems. Nature. 2007; 446:782-786. [PubMed: 17429397]

49. Strümpfer J, Şener M, Schulten K. How Quantum Coherence Assists Photosynthetic LightHarvesting. J Phys Chem Lett. 2012; 3:536-542. [PubMed: 22844553]

50. Orrit M, Bernard J. Single Pentacene Molecules Detected by Fluorescence Excitation in a ParaTerphenyl Crystal. Phys Rev Lett. 1990; 65:2716-2719. [PubMed: 10042674]

51. Pisoni DS, et al. Symmetrical and Asymmetrical Cyanine Dyes. Synthesis, Spectral Properties, and BSA Association Study. J Org Chem. 2014; 79:5511-5520. [PubMed: 24845528]

52. Karstens T, Kobs K. Rhodamine-B And Rhodamine-101 as Reference Substances for Fluorescence Quantum Yield Measurements. JPhys Chem. 1980; 84:1871-1872.

53. Texier I, et al. Cyanine-loaded lipid nanoparticles for improved in vivo fluorescence imaging. $\mathbf{J}$ Biomed Opt. 2009; 14:054005. [PubMed: 19895107]

54. Santra K, et al. What Is the Best Method to Fit Time-Resolved Data? A Comparison of the Residual Minimization and the Maximum Likelihood Techniques as Applied to Experimental Time-Correlated, Single-Photon Counting Data. J Phys Chem B. 2016; 120:2484-2490. [PubMed: 26865463]

55. Lakowicz, JR. Principles of Fluorescence Spectroscopy. Springer; 2006.

56. Zhao QL, Young IT, de Jong JGS. Photon budget analysis for fluorescence lifetime imaging microscopy. J Biomed Opt. 2011; 16:086007. [PubMed: 21895319] 


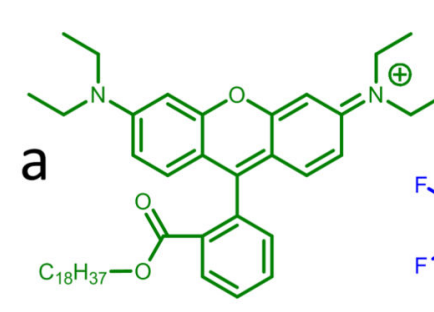

R18<smiles>Fc1c(F)c(F)c(B(c2c(F)c(F)c(F)c(F)c2F)c2c(F)c(F)c(F)c(F)c2F)c(F)c1F</smiles>

F5-TPB
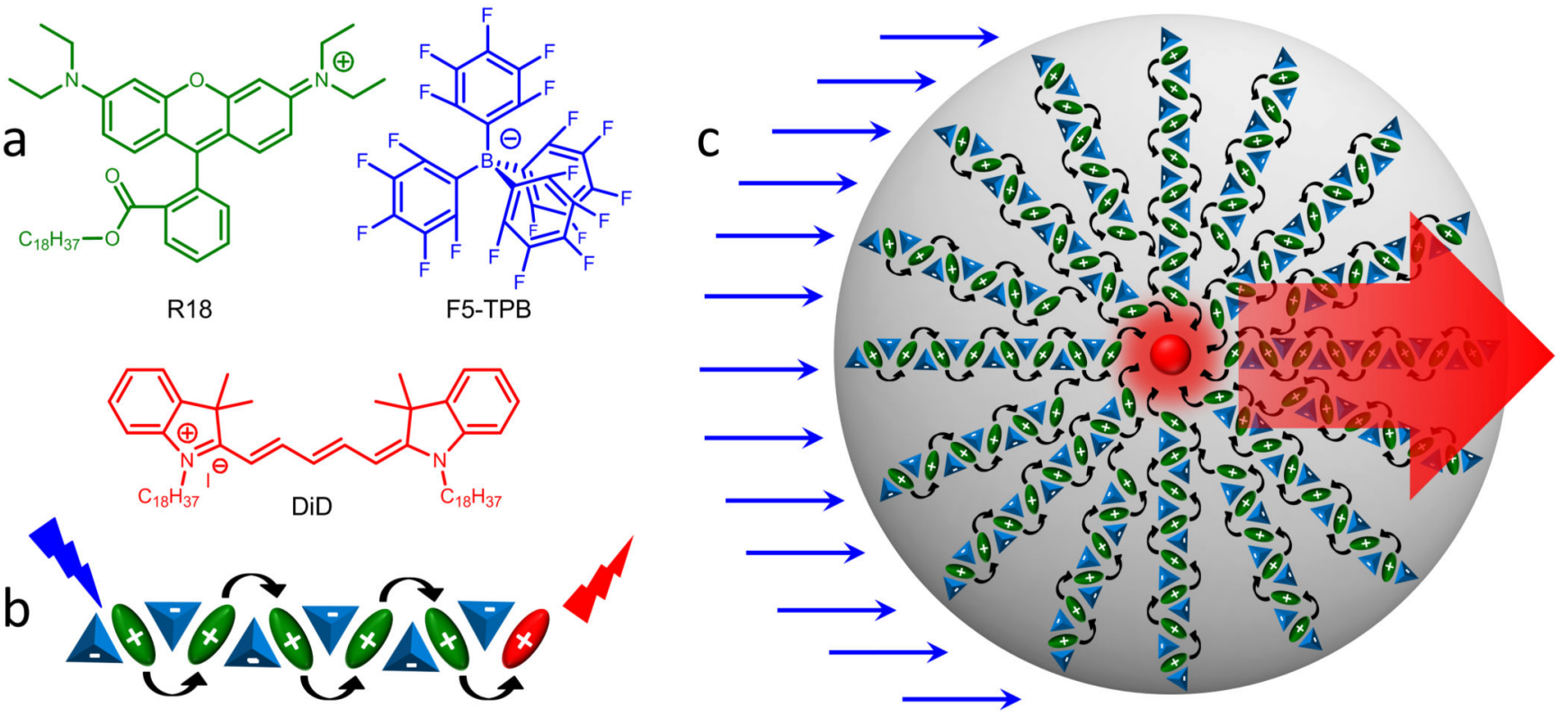

Figure 1. Concept of organic nano-antenna.

(a) Chemical structures of the donor dye rhodamine B octadecyl ester (R18) and its counterion tetrakis(pentafluorophenyl)borate (F5-TPB) and of the acceptor Cy5 dye (DiD). (b) Short-range ordering of R18 cations (green) by the F5-TPB counterion (blue) inside the PMMA-MA matrix prevents dye aggregation and leads to short interfluorophore distances and ultrafast EET with subsequent FRET to a single acceptor molecule (red). (c) Schematic presentation of the giant light harvesting nano-antenna concept inside polymer NPs. For simplicity, the donor dyes are presented well-organized around the acceptor placed in the centre of the particle. 

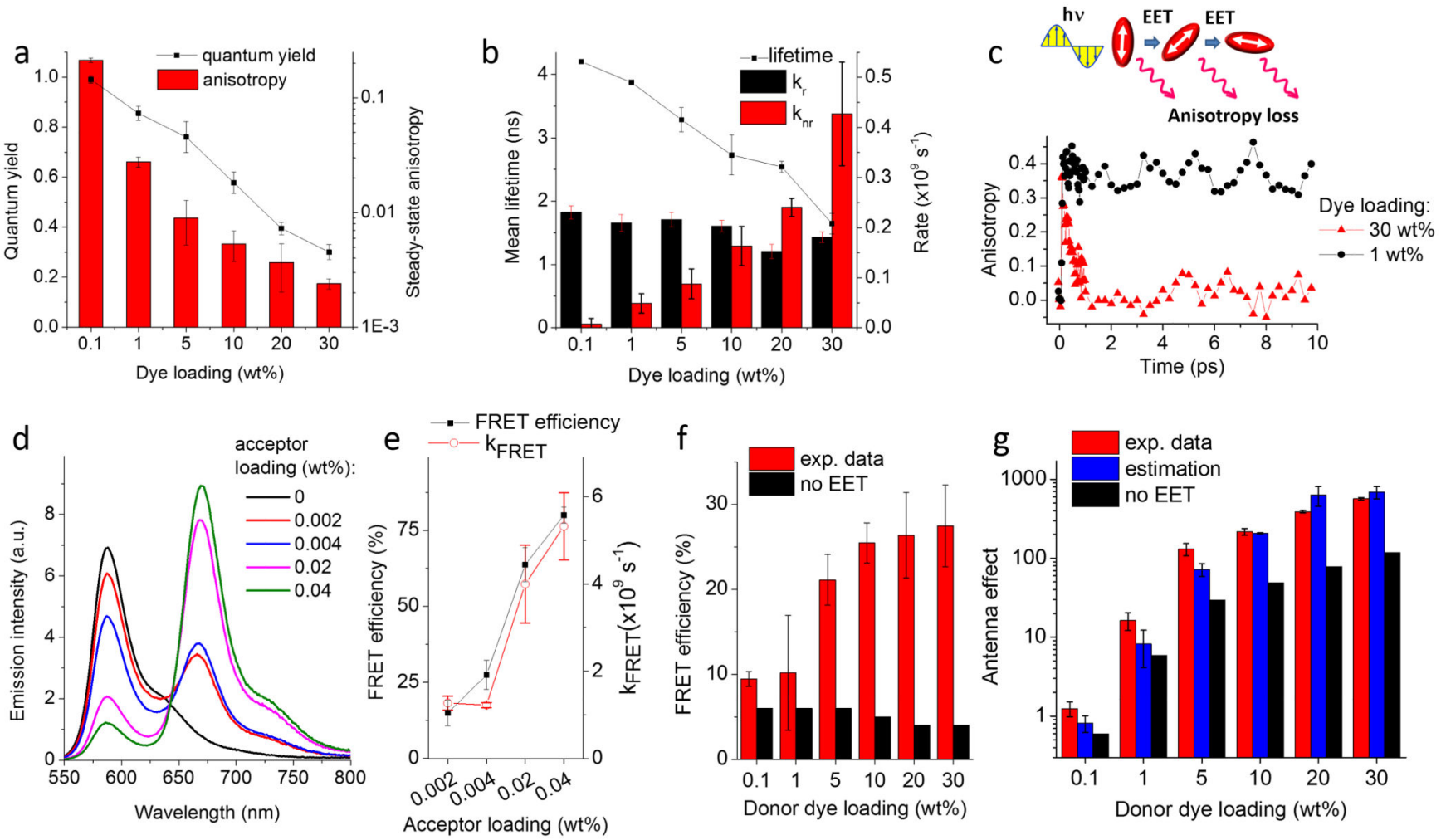

Figure 2. Spectroscopic characterization of organic nano-antennas.

(a) Fluorescence quantum yield and steady-state anisotropy and (b) mean fluorescence lifetime, radiative $\left(\mathrm{k}_{\mathrm{r}}\right)$ and non-radiative $\left(\mathrm{k}_{\mathrm{nr}}\right)$ rate constants for NPs at different loading of R18/F5-TPB. (c) Scheme of fluorescence anisotropy loss due to EET within randomly oriented fluorophores. Emission anisotropy decay measured at $580 \mathrm{~nm}$ with a 60 -fs probe beam for NPs loaded at 1 and $30 \mathrm{wt} \%$ of R18/F5-TPB. (d) Emission spectra of FRET NPs loaded with different amounts of the acceptor dye (DiD) while keeping the same amount of the R18/F5-TPB donor (30 wt \%). The emission intensity was normalized to the same absorbance of the donor. (e) FRET efficiency and FRET rate calculated from the emission decay of acceptors. NPs contained $30 \mathrm{wt} \%$ donor and varied concentrations of acceptor. (f) Experimental FRET efficiency for FRET NPs with varied donor but constant acceptor (0.004 wt $\%$ ) concentration and the calculated one assuming no EET. (g) Antenna effect measured from the excitation spectra, its estimated values based on the observed FRET efficiency and theoretical estimation assuming no EET for FRET NPs with varied donor but constant acceptor $(0.004 \mathrm{wt} \%)$ concentration. Error bars in $(\mathrm{a}, \mathrm{e}, \mathrm{f})$ are s.e.m. $(\mathrm{n}=3)$. 
a

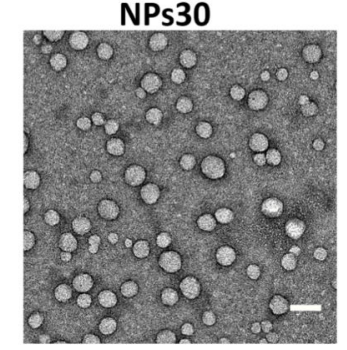

NPs45

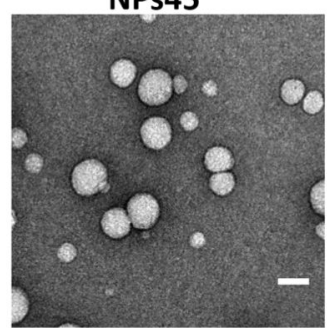

NPs60

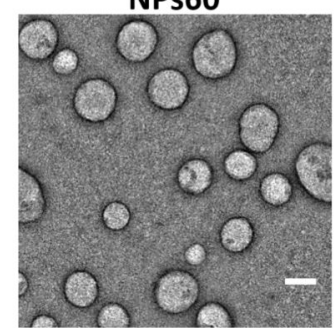

NPs65
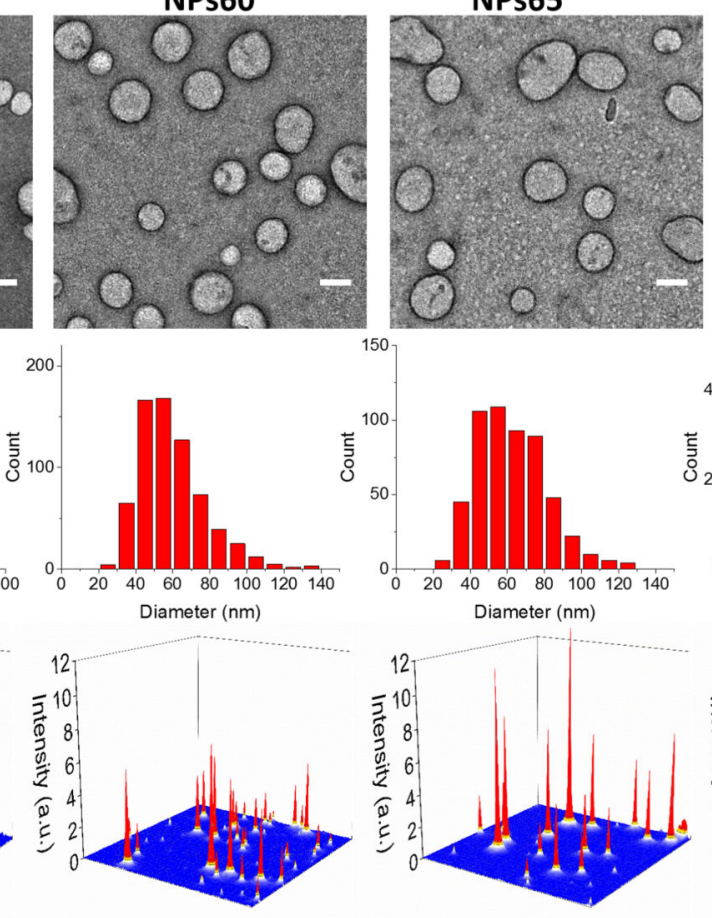
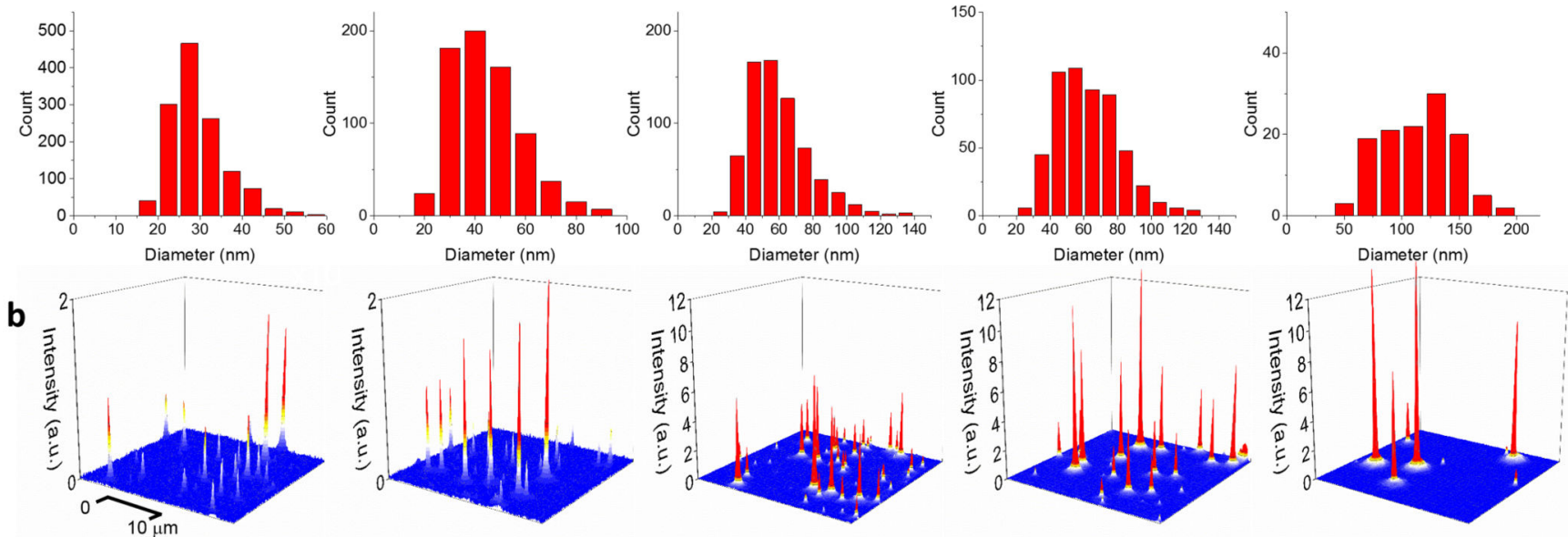

Figure 3. TEM and fluorescence microscopy images of individual nano-antennas.

(a) TEM images and size histograms of NPs containing $30 \mathrm{wt} \%$ R18/F5-TPB prepared at different $\mathrm{pH}$. Scale bar, $50 \mathrm{~nm}$ (b) 3D representation of wide-field fluorescence microscopy images of these NPs under illumination of a $532 \mathrm{~nm}$ laser with power density of $0.1 \mathrm{~W} \mathrm{~cm}^{-2}$. The integration time was $30.53 \mathrm{~ms}$. 

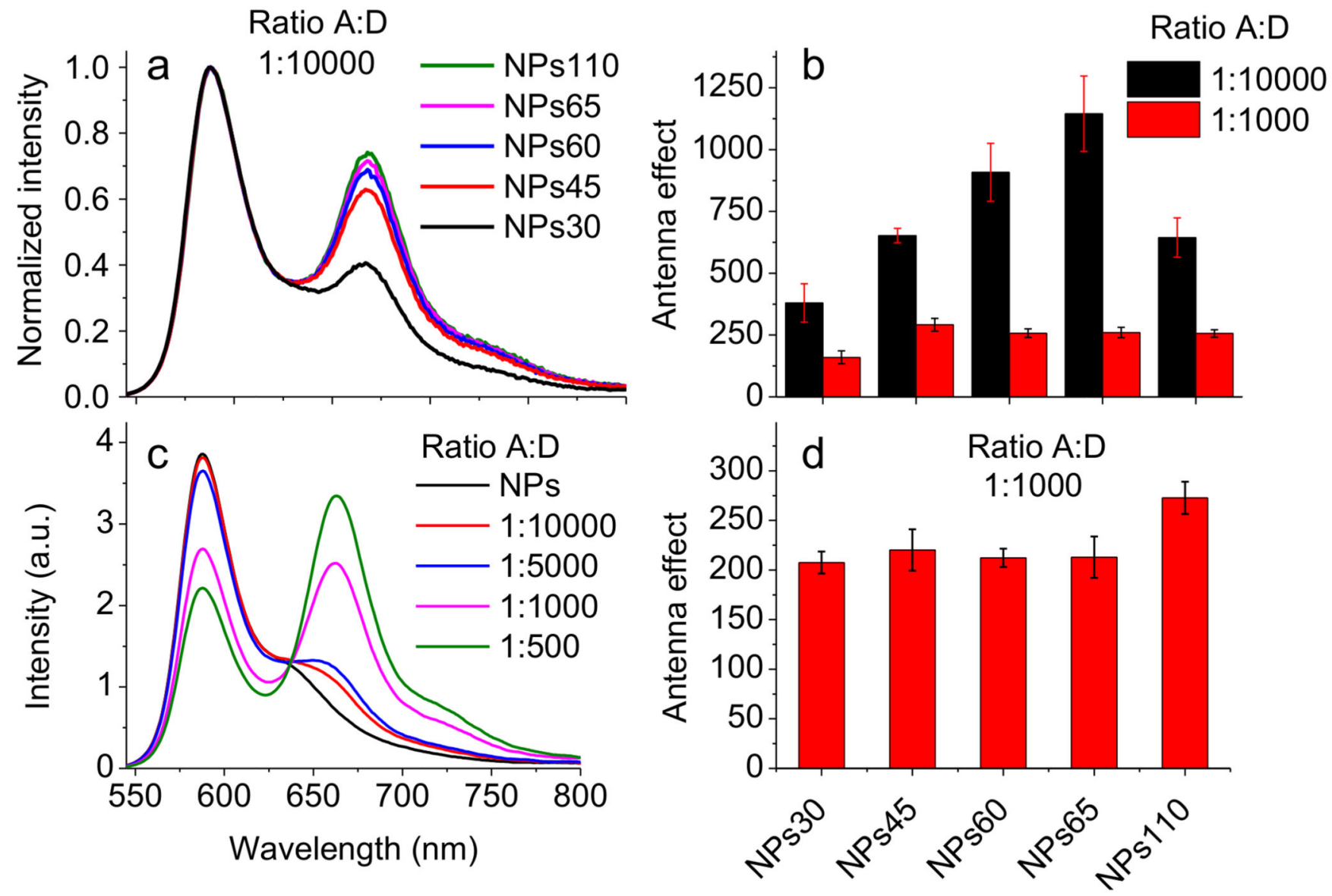

Figure 4. Fluorescence spectra and antenna effect of FRET NPs bearing acceptor inside (a, b) or at the surface $(c, d)$ of NPs.

(a) Fluorescence spectra of PMMA-MA FRET NPs of different sizes loaded with $30 \mathrm{wt} \%$ of R18/F5-TPB and DiD with donor/acceptor ratio 10,000:1. (b) Antenna effect of NPs of different size loaded with $30 \mathrm{wt} \%$ of R18/F5-TPB and DiD at ratios 10,000:1 or 1,000:1. (c) Spectra of NPs45 loaded with $30 \mathrm{wt} \%$ of R18/F5-TPB upon addition of acceptor molecule Cy5-C2. (d) Amplification of acceptor emission (antenna effect) of Cy5-C2 adsorbed on the surface of NPs of different sizes loaded with $30 \mathrm{wt} \%$ of donor at an acceptor concentration corresponding to a donor/acceptor ratio of 1,000:1. Error bars represent s.e.m. $(n=3)$. 


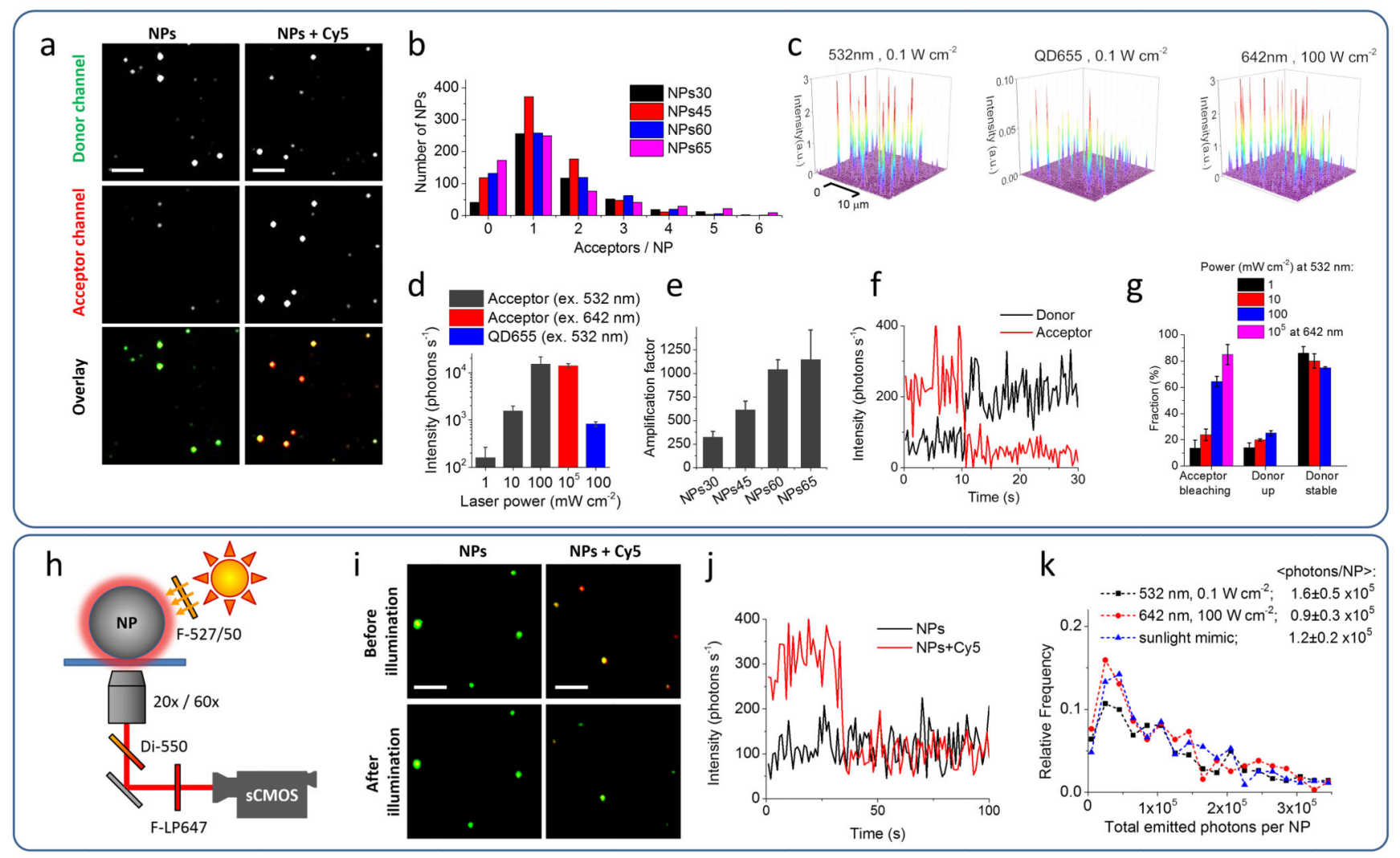

Figure 5. Single-particle evaluation of nano-antennas.

(a) Wide-field fluorescence microscopy images of NPs. NPs60 containing $30 \mathrm{wt} \%$ R18/F5TPB without (left panels) and with $\sim 1.3$ Cy5 (DiD) acceptors per NP (donor:acceptor ratio is 10,000:1, right panels). The laser excitation was at $532 \mathrm{~nm}$ (power density $0.1 \mathrm{~W} \mathrm{~cm}^{-2}$ ). Both channels are represented at the same intensity scale. Overlay images represent false colour composite of donor (green) and acceptor (red) channels. Scale bar, $3 \mu \mathrm{m}$. (b)

Histogram of the number of acceptors per NPs calculated from single-molecule photobleaching events using excitation at $642 \mathrm{~nm}\left(100 \mathrm{~W} \mathrm{~cm}^{-2}\right)$. (c) $3 \mathrm{D}$ representation of wide-field TIRF images of acceptor emission from NPs60 containing 1.3 Cy5 acceptors per NP under illumination at $532 \mathrm{~nm}\left(0.1 \mathrm{~W} \mathrm{~cm}^{-2}\right)$, under direct excitation of the acceptor at $642 \mathrm{~nm}\left(100 \mathrm{~W} \mathrm{~cm}^{-2}\right)$ and of QD655 under illumination at $532 \mathrm{~nm}\left(0.1 \mathrm{~W} \mathrm{~cm}^{-2}\right)$. The integration time was $30.53 \mathrm{~ms}$. (d) Absolute fluorescence intensity of the acceptors in single NPs60 excited at 532 or $642 \mathrm{~nm}$ in comparison to that of QDs655. (e) Amplification factor of acceptor emission for antennas of different sizes with 1-2 acceptors per NP. (f)

Representative single-particle trace excited at $532 \mathrm{~nm}\left(1 \mathrm{~mW} \mathrm{~cm}^{-2}\right)$. (g) Fraction of single acceptor bleaching events and the response of donor dyes to these events. (h-k) Scheme of experimental setup and obtained results, using excitation that mimics direct sunlight. (i) Single-particle FRET microscopy with overlaid donor (green) and acceptor (red) channels under sunlight excitation mimics using NPs60 containing $\sim 1.3$ Cy5 per NP before and after 5-min illumination. Scale bar, $3 \mu \mathrm{m}$. (j) Single-particle traces at the acceptor channel for NP60 without and with Cy5 acceptor. (k) Histogram of the total photons emitted before 
photobleaching for the single acceptors excited directly or through NPs60 nano-antenna. All errors are s.e.m. $(n \geq 3)$. 
Table 1

Size, spectroscopic and single-particle properties of PMMA-MA NPs encapsulating 30 wt\% of R18/F5-TPB prepared at varied $\mathrm{pH}$.

\begin{tabular}{|c|c|c|c|c|c|c|c|c|c|}
\hline Sample & $\mathrm{pH}^{a}$ & Size, DLS (nm) $b$ & Size, TEM $(\mathbf{n m})^{c}$ & Mean lifetime, $\mathrm{ns}^{d}$ & $\mathbf{Q Y}^{e}$ & Anisotropy & Donors/NP $f$ & $\begin{array}{l}\mathrm{SPB}^{g} \\
\text { estim. }\end{array}$ & $\begin{array}{l}\text { SPB } h \\
\text { exp. }\end{array}$ \\
\hline NPs30 & 9.0 & $36 \pm 1$ & $29 \pm 1$ & $1.9 \pm 0.3$ & $0.31 \pm 0.04$ & 0.001 & 1,700 & 1 & 1 \\
\hline NPs45 & 7.4 & $67 \pm 2$ & $44 \pm 2$ & $1.6 \pm 0.2$ & $0.30 \pm 0.03$ & 0.0028 & 5,900 & 3 & 5 \\
\hline NPs60 & 6.7 & $105 \pm 5$ & $58 \pm 2$ & $1.7 \pm 0.1$ & $0.28 \pm 0.03$ & 0.0025 & 13,000 & 7 & 12 \\
\hline NPs65 & 6.5 & $144 \pm 6$ & $63 \pm 4$ & $1.7 \pm 0.1$ & $0.27 \pm 0.03$ & 0.0024 & 17,000 & 9 & 20 \\
\hline NPs110 & 5.8 & $231 \pm 16$ & $113 \pm 6$ & $1.7 \pm 0.2$ & $0.29 \pm 0.03$ & 0.0029 & 99,000 & 54 & 31 \\
\hline
\end{tabular}

${ }^{a}$ After preparation, NPs were diluted in $\mathrm{pH} 7.4$ buffer.

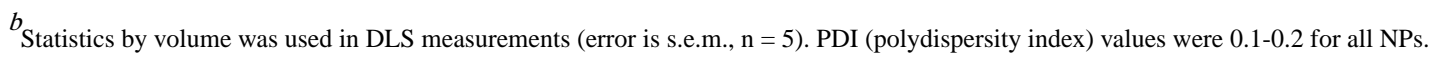

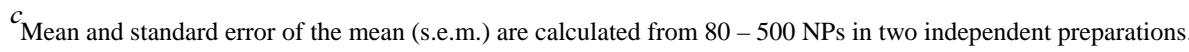

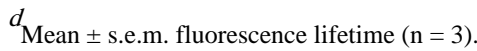

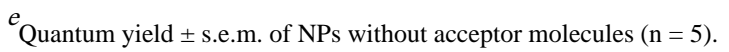

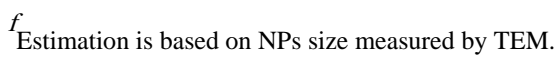

${ }^{g}$ Estimated single-particle brightness (SPB) normalized to NPs30 based on QY and size of NPs measured by TEM.

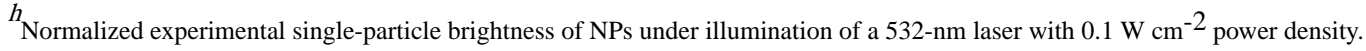

